Le concept de réseau dans l'univers ferroviaire. Conclusions de la journée, par le professeur Maurice Wolkowitsch

\title{
Maurice Wolkowitsch
}

\section{CpenEdition}

\section{Journals}

Édition électronique

URL : https://journals.openedition.org/rhcf/882

DOI : $10.4000 /$ rhcf.882

\section{Éditeur}

Rails \& histoire

\section{Édition imprimée}

Date de publication : 1 juin 2008

Pagination : 139-143

ISSN : 0996-9403

\section{Référence électronique}

Maurice Wolkowitsch, « Le concept de réseau dans l'univers ferroviaire. Conclusions de la journée, par le professeur Maurice Wolkowitsch », Revue d'histoire des chemins de fer [En ligne], 39 | 2008, mis en ligne le 01 juin 2011, consulté le 22 avril 2022. URL : http://journals.openedition.org/rhcf/882 ; DOI https://doi.org/10.4000/rhcf.882 


\section{Le concept de réseau dans l'univers ferroviaire. Conclusions de la journée, par le professeur Maurice Wolkowitsch*}

Cette journée d'étude sur le concept de réseau dans l'univers ferroviaire me semble avoir répondu à notre attente, j’en veux pour preuve l'assiduité de l'auditoire qui ne s'est pas démentie.

Les intervenants dans leur communication ou dans les débats ont abordé le sujet sous tous ses aspects, prenant en compte les divers modes de transport ferroviaire malgré l'absence du tramway, à toutes les échelles, ville, État, Europe. Des conceptions variées se sont exprimées, ce qui nous a valu une grande diversité de méthodes dans la recherche d'une définition de la notion de réseau. L'étude des étapes de la formation des réseaux a permis de voir les résultats des périodisations établies par les historiens, mais aussi par l'application de la théorie des graphes. M. Bouley a opposé deux systèmes, l'un vectoriel, correspondant à des nécessités économiques et dont la construction suppose le profit, l'autre à un réseau voulu dans une perspective de cohésion et action politiques et correspondant davantage à une conception administrative.

Cette richesse ne saurait nous faire oublier qu'il reste beaucoup à faire ; de multiples pistes de recherche s'ouvrent devant les membres de l'Association, nous en évoquerons quatre.

\footnotetext{
* Notes de la rédaction (les notes de l'auteur sont placées à la fin de son texte) : Extrait de la publication des Actes de la première journée scientifique de l'AHICF (11 octobre 1989), «Le concept de réseau dans l'univers ferroviaire ", Revue d'bistoire des chemins de fer $\mathrm{n}^{\circ} 2$ (printemps 1990), 284 pages, sous la direction de Maurice Wolkowitsch et Paul Claval, dont le sommaire figure en annexe.
} 
1. Toutes les études conçues dans le sens d'une application de la théorie des graphes méritent d'être poursuivies et précisées. Mme Dancoisne a montré quelles applications on pouvait en tirer : il faudrait multiplier les comparaisons entre réseaux régionaux, nationaux pour aboutir peut-être à une typologie d'état de la desserte d'un territoire donné. Compte tenu de la complémentarité entre certains modes de transport, ne faudrait-il pas pousser plus loin les analyses en combinant par exemple les réseaux de chemin de fer et d'autobus sur un territoire donné ? Enfin la comparaison entre les résultats obtenus par les historiens et les utilisateurs de la théorie des graphes pour définir les dates charnières dans les étapes de formation des réseaux aurait intérêt à être affinée.

2. M. Peny a évoqué le rôle de la station comme intermédiaire entre la ville et le réseau de métro. L'idée reste valable pour la gare de chemin de fer. René Clozier a consacré en 1940 sa thèse à la Gare du Nord(1); il en a retracé l'histoire, les agrandissements successifs en liaison avec le développement des trafics de marchandises et de banlieue. La gare est aussi l'interface entre transport urbain et transport ferroviaire. La gare traduit dans son architecture la puissance de la compagnie ; elle est un élément de perturbation mais aussi de structuration dans un quartier, elle joue un rôle dans l'aménagement. Au moment où de nouvelles gares apparaissent (Lyon
Part-Dieu, gares des TGV...) ou d'autres sont restructurées avec les quartiers alentours, des études consacrées à ces questions enrichiraient utilement nos connaissances. Même la situation juridique des gares pourrait être évoquée : M. Poinssot, directeur Commercial Fret de la SNCF, à un jury de thèse se demandait si les chambres de commerce qui gèrent les aéroports ne se sentiraient pas davantage concernées par le chemin de fer, si elles étaient associées à la gestion des gares.

3. L'histoire de l'évolution des réseaux offre des pistes de recherche ouvrant de larges possibilités. L'étude du développement du réseau a donné lieu à d'innombrables publications et peut en alimenter bien d'autres. Nous souhaitons attirer l'attention sur la rareté des recherches consacrées à la contraction des réseaux ; il convient cependant de signaler l'étude globale qui en a été présentée à propos des réseaux allemand, anglais et français par E. Auphan dans sa thèse(2).

L'ouverture d'esprit et l'appui de deux ingénieurs de la région SudOuest, MM. Menu et Rousselet, m'avaient permis d'accéder, il y a une quarantaine d'années, aux dossiers de fermeture de ligne et aux plans départementaux de transport, documents essentiels pour la période 1938-1952. Un demi-siècle s'est écoulé, il s'agit bien d'histoire : le propos n'est pas de juger la politique ferroviaire du gouvernement et de la SNCF, mais d'en définir les 
objectifs et les conditions d'application. Quelles orientations de recherche suggérer?

La politique de contraction du réseau est voulue par le gouvernement et exécutée par la SNCF qui, dans ses sphères dirigeantes, y est acquise : elle correspond à la volonté d'une gestion plus économe, mais aussi au souhait de transférer au transport routier une part du trafic ferroviaire. L'encouragement de l'État se traduit dans les avantages financiers promis aux départements présentant un plan de transport « raisonnable » avant le $1^{\text {er }}$ juillet 1938 : $150000 \mathrm{~F}$ plus $3000 \mathrm{~F}$ par an et kilomètre de ligne fermée ou partiellement exploitée. Les résultats de ces mesures et les dépenses correspondantes n'ont jamais fait l'objet d'analyses d'ensemble.

Les conseillers généraux dans leur immense majorité (font exception ceux qui sont très proches du gouvernement) sont hostiles aux fermetures et le manifestent dans leurs délibérations ; il en va de même au niveau municipal ; la presse locale consacre beaucoup d'articles à la question des transports avec des points de vue plus nuancés que ceux des élus. Ces diverses formes d'expression de l'opinion publique, si soigneusement rassemblées et conservées lors de la construction des réseaux, sont étrangement absentes des dossiers bien minces de fermeture de ligne parce qu'elles ne vont pas dans le sens souhaité. Collecter cette documentation aurait, me semble-t-il, un grand intérêt.
L'action des préfets mérite l'attention : manœuvres pour placer un conseil général rétif devant le vote d'une assemblée voisine, plus compréhensive ; un vote négatif (le refus de la fermeture de lignes) du premier devient impossible, les lignes s'achevant en cul-de-sac à la limite du département ; utilisation d'une lettre de la direction de la SNCF réclamant une décision urgente du conseil général pour éviter de lourds investissements indispensables en cas de maintien en activité de lignes condamnées ; cette argumentation se retrouve comme un leitmotiv pour les lignes concernées de chaque département, au point qu'on s'interroge sur sa réalité, ou alors il faut admettre que le réseau ait été bien délaissé ? Transmission du plan de transport avec une note confidentielle d'un préfet signalant les entreprises routières demandant des compensations pour des services jamais exploités. Tout cela peut éclairer les conditions dans lesquelles la politique de coordination des transports a débuté.

Les demandes de sanctions contre des hommes de terrain jugeant la politique de fermeture de ligne contraire à l'intérêt général et local posent beaucoup de questions; nous en avons trouvé à propos d'un ingénieur des Ponts et Chaussées qui avait voté au comité technique départemental de la Haute-Vienne contre la fermeture de deux lignes et d'un ingénieur de la SNCF qui avait communiqué à des conseillers généraux des arguments à 
opposer à ceux de la SNCF. Le phénomène s'est-il souvent reproduit? Y a-t-il eu ou non des sanctions ? Des agents de l'État ou d'une entreprise publique peuvent-ils exprimer une opinion personnelle sur des questions qu'ils connaissent parfaitement? Jusqu'où peut aller la liberté d'expression et jusqu'où s'impose le devoir de réserve ? Les problèmes de rapports au sein de l'entreprise apparaissent à cette occasion pleinement posés.

L'analyse des plans de transport montre la SNCF et les transporteurs routiers échanger des kilomètres voyageurs d'un bout de la France à l'autre (arrêt de la concurrence routière entre Perpignan et Banyuls en échange de services de remplacement de lignes fermées dans l'Indre), sans aucune analyse des conséquences pour la population.

Comparer la desserte d'un département pour les marchandises et les voyageurs avant et après l'adoption des mesures préconisées serait riche d'enseignements. Quels ont été les effets sur le rayonnement des villes et bourgs, y a-t-il eu des modifications dans l'aire d'approvisionnement et dans les marchés des entreprises agricoles, industrielles et commerciales ? Autant de questions auxquelles la recherche n'a guère apporté de réponse, alors que les effets positifs du chemin de fer ont alimenté une littérature abondante. Sans doute l'autocar et le camion étaient-ils prêts à prendre la relève, mais comment ont réagi concrètement, localement les habitants, des habitudes de déplacements ontelles été modifiées?

Pour répondre à ces interrogations, quelles sont aujourd'hui les sources disponibles, sont-elles accessibles aisément?

4. La communication de M. Bouley nous a rappelé le rôle politique des réseaux ferrés ; la cohésion des États a conduit les gouvernements à s'intéresser à leurs tracés. Aujourd'hui le réseau des lignes à grande vitesse ne doit-il pas apparaitre comme un instrument capable de favoriser et forger l'unité de l'Europe ? La réflexion géopolitique s'inscrit aussi dans notre horizon de recherche.

La richesse des débats montre qu'il reste beaucoup à faire ; professionnels, qui pourraient peut-être plus aisément accéder à certaines sources, et chercheurs n'ont plus qu'à se mettre au travail.

\section{NOTES}

1. Clozier, René, La Gare du Nord, Paris, Baillère, 1940, 294 p., 110 fig.

2. Auphan, Etienne, "Obsolescence ou renaissance des réseaux ferrés pour le transport de voyageurs en Europe occidentale? (France, Grande-Bretagne, Allemagne fédérale) », Thèse d'État, Université d'Aix-Marseille II, décembre 1989, exemplaires dactylographiés, 2 tomes $(796+694$ p.), 367 fig., 45 phot.**

** Thèse partiellement publiée depuis lors sous le titre: Quel avenir pour les réseaux ferrés d Europe occidentale? (Paris, éd. du CNRS, 1991, 204 pages). 


\section{REVUE D'HISTOIRE DES CHEMINS DE FER}

\section{Numéro 2 - printemps 1990}

\section{Le concept de réseau dans l'univers ferroviaire}

\section{Editorial}

Le concept de réseau dans l'univers ferroviaire, par MM. Maurice Wolkowitsch et Paul Claval, membres du Comité scientifique de l'AHICF.

\section{Communications}

Allocution de M. Maurice Wolkowitsch

Evolution du réseau et hiérarchie des voies ferrées, par Etienne Auphan.

Des saint-simoniens à Léon Lalanne : projets, thèses et controverses à propos de l'organisation des réseaux ferroviaires, par Georges Ribeill.

Discussions

Réseaux ferroviaires et relations interrégionales en France, par Jean Varlet.

Utilisation de la théorie des graphes pour l'analyse des réseaux ferroviaires, par Pascale Dancoisne.

La ligne $\mathrm{C}$ du métro lyonnais : un réseau "multi-services" ?, par Jean-Marc Offner et Franck Scherrer.

Discussions

De la ligne au réseau à grande vitesse : le concept de réseau dans l'émergence des grandes vitesses en France, par Jean-Michel Fourniau.

Le réseau à très grande vitesse européen se construit, par Jean Bouley.

Discussions

Entre ville et réseau : la station de métro, par André Pény.

Discussions

Allocution de M. François Caron, Secrétaire général de l'AHICF.

Conclusion de la Journée, par Maurice Wolkowitsch. 
